\title{
Visualizing State Identification in Auto-Regressive Hidden Markov (ARHMM) Models With the Forward and Backward Algorithms Using Excel
}

\author{
William. H. Laverty ${ }^{1}$ \& Ivan. W. Kelly ${ }^{2}$ \\ ${ }^{1}$ Department of Mathematics and Statistics, University of Saskatchewan, Saskatoon, Canada \\ 2 Professor Emeritus, Department of Educational Psychology \& Special Education, University of Saskatchewan, \\ Saskatoon, Canada \\ Correspondence: W. H. Laverty, Department of Mathematics and Statistics, 142 McLean Hall, University of \\ Saskatchewan, Saskatoon, SK, S7N-5E6, Canada.
}

Received: June 22, 2019 Accepted: July 15, 2019 Online Published: August 6, 2019

doi:10.5539/ijsp.v8n5p25 URL: https://doi.org/10.5539/ijsp.v8n5p25

\begin{abstract}
Earlier articles, Laverty, Miket, Kelly (2002c), Laverty and Kelly (2019) used Excel to simulate Hidden Markov models and calculate the probabilities of the unknown states using the forward and backward algorithms (Rabiner, 1989). In those articles, independence between observations in each state were assumed. In many situations, however, the assumption of independence within states cannot be made. A more appropriate model for the data in this case would be an Autoregressive Hidden Markov model which accounts for serial correlation within states. In this article, a two-state ARHMM will be simulated with the forward-backward algorithm used to calculate conditional state probabilities given the observed data.
\end{abstract}

Keywords: auto-regressive Hidden Markov model, forward algorithm, backward algorithm, state probabilities, Excel

\section{Introduction}

Hidden Markov models (HMMs) are a collection of widely used statistical models. These models are applicable when studying a process that goes through a sequence of states. The states are unseen (hidden) but what is observed is data from each state. For example, HMMs have been used to model heart rate variability (Walker 11, 2011), to model financial data (Mamon \& Elliott, 2007), (Genon-Catalot, Jeantheau \& Laredo, 2000), and to model residuals in regression (Laverty, Miket \& Kelly, 2002a), Laverty, Miket \& Kelly (2002b). Applications of Hidden Markov Models are possible whenever we have data collected over time. The traditional time series models (AR, MA, ARMA etc.) assume that the process generating the data is constant over a single state throughout the entire time period. However, when the data sequence is over a long period of time it is likely that there are changes in the states that are generating the data. In a Hidden Markov Model, the observations could be assumed to be independent when the state is constant. Alternatively, when the state is constant the observations might be correlated and better modelled by an Autoregressive (AR) time series. This leads to the Autoregressive Hidden Markov (ARHMM) model described in Bing-hwang \& Rabiner, (1986). Applications of the ARHMM models have been used by Stanculescu, Williams, \& Freer, (2014) and Tang (2004).

An important problem is to identify the hidden states that have generated the observed data. A common approach is to calculate the probability of the HMM (or ARHMM) being in a certain state at a certain time given either the data up to that time or the complete set of data using the forward-backward algorithm described in Rabiner (1989) (for HMM) or Stanculescu, Williams, \& Freer, (2014) and Tang, (2004) (for ARHMM). This calculation assumes the parameters of the model (HMM or ARHMM) are known. If the parameters of the model are unknown they can be estimated using the techniques described in Bing-hwang, and Rabiner, (1986) or Rabiner, (1989).

\section{Autoregressive Hidden Markov Models}

An autoregressive hidden Markov model will consist of a sequence of states $X_{1}, X_{2}, \ldots, X$ together with a sequence of observations $Y_{1}, Y_{2}, \ldots, Y_{T}$. We assume that the number of states (possible values of each $X_{i}$ ) is a finite number, $m$. The states can be represented by the integers $1,2, \ldots, m$. The states are not observed. 
The observations $Y_{1}, Y_{2}, \ldots, Y_{T}$ are observed and could be vectors of dimension $k$. In this paper $k=1$. The distribution of the observation $Y_{t}$ at time $t$ depends on the previous two states $X_{t}$ and $X_{t-1}$ of the Markov process and the previous observation $Y_{t-1}$. In this paper we are assuming that there are only two states and that the distribution of $Y_{t}$ given $X_{t}=i, X_{t-1}=j, Y_{t-1}=y_{t-1}$ is the Normal distribution with adjusted mean, $\mu_{i}+\beta_{i}\left(\frac{y_{t-1}-\mu_{j}}{\sigma_{j}}\right)$ and standard deviation $\sigma_{i}$. The parameters of an ARHMM process are $\mu_{i}, \sigma_{i}, \beta_{i}(i=1,2)$.

In addition, the parameters of the Markov process model are the initial state probabilities,

$$
\pi_{i}=\operatorname{Pr}\left(X_{1}=i\right) \quad i=1,2, \quad \ldots, m
$$

and the transition probability matrix $\Gamma=\left(\gamma_{\mathrm{ij}}\right)$. This is an $\mathrm{m} \times \mathrm{m}$ matrix, with element $\gamma_{\mathrm{ij}}$ being the probability of a transition into state $j$ starting from state $i$.

i.e.

$$
\gamma \mathbf{\imath} \varphi=\Pi \rho(\Xi \tau=\varphi \lambda \Xi \tau-1=\imath),
$$

where $t$ denotes time. These two choices allow us to construct a sequence of states (known also as the Markov chain) $X_{1}, X_{2}, \ldots, X_{T}$ constituting the hidden part of a hidden Markov model.

When the Markov chain is in state $i$, at time $t$ and state $j$ at time $t-1$ it emits an observed signal $Y_{t}$, a continuous random variable (or random vector) with distribution conditional on the current state $i$. the previous state $j$ and the observation at time $t-1, Y_{t-1}$

\section{Simulation of an Autoregressive Hidden Markov Model With Normal Observations in Excel}

Uniform random variates on [0,1] can be generated in Excel with the function "RAND()". The generation of random variates with a Normal distribution with mean and standard deviation, can be carried out using the inverse-transform method (Fishman (2)). Namely $Y=F^{-1}(U)$ where $F(u)$ is the desired cumulative distribution of $Y$ and $U$ has a uniform distribution on [0,1]. In Excel this is achieved for the Normal distribution (mean, standard deviation) with the function "NORMINV(RAND (), ,)."

To simulate an Autoregressive Hidden Markov model with $m=2$ states and normal observations with mean $\mu_{i}$, standard deviation $\sigma_{I}$ and autoregressive parameter $\beta_{i}$ when the Markov process is in state $i$ we again need to determine the sequence of states then generate the observations from those states.

Initially we will store the parameters of the model in various cells of the excel spread sheet. For example the transition probabilities $\gamma_{i j}(i=1,2 ; j=1,2)$ will be stored in the cells B3:C4, the initial probabilities $\pi_{i}(i=1,2)$ will stored in cells B9:C9, and the parameters of ARHMM the normal distribution $\left(\mu_{i}, \sigma_{i}, \beta_{i}\right) i=1,2$ will be stored in cells H3:J4.

The next step is to generate the sequence of states. We generate the first state by determining if a uniform random variate $U$ is above or below $\pi_{1}$. This is achieved by placing the formula " $\operatorname{IF}(\operatorname{RAND}()<\mathrm{B} 9,1,2)$ " in cell C13. We now generate the following sequence of states determining if a uniform random variate $U$ is above or below $\gamma_{i 1}$. This is achieved by placing the formula " $\operatorname{IF}(\mathrm{OR}(\mathrm{AND}(\mathrm{C} 13=1, \operatorname{RAND}()<\mathrm{B} \$ 3), \operatorname{AND}(\mathrm{C} 13=2, \operatorname{RAND}()<\mathrm{B} \$ 4)), 1,2)$ " in cell C14. This formula can now be copied down to generate as many states as desired (In this paper we generate 200 states and observations). The final step is to generate normal observations with mean $\mu_{i}+\beta_{i}\left(\frac{y_{t-1}-\mu_{j}}{\sigma_{j}}\right)$ and standard deviation $\sigma_{i}$ at each time point, $t$, when the process is in state $i$ at time $t$ and state $j$ at time $t-1$. To obtain an observation with the above properties we would compute $y_{t}=\mu_{i}+\beta_{i}\left(\frac{y_{t-1}-\mu_{j}}{\sigma_{j}}\right)+\sigma_{i} u_{t}$ where $u_{t}$ is a $N(0,1)$ random variate. This is achieved by pasting the formula

“=VLOOKUP(C14,G\$4:H\$5,2)+VLOOKUP(C14,G\$4:J\$5,4)*(B13-VLOOKUP(C13,G\$4:H\$5,2))/VLOOKUP( C13,G\$4:I\$5,3)+VLOOKUP(C14,G\$4:I\$5,3)*NORMSINV(RAND())"'into cell B13.

Note: VLOOKUP(C14,G\$4:H\$5,2) obtains the value $\mu_{i}$

VLOOKUP(C13,G\$4:H\$5,2) obtains the value $\mu_{j}$

VLOOKUP(C14,G\$4:I\$5,3) obtains the value $\sigma_{i}$

VLOOKUP(C13,G\$4:I\$5,3) obtains the value $\sigma_{j}$, and

VLOOKUP(C14,G\$4:J\$5,4) obtains the value $\beta_{i}$. 
Again, this formula can now be copied down to generate the complete set of data. Below is a copy of the spreadsheet with graphs of the data sequence and the state sequence.

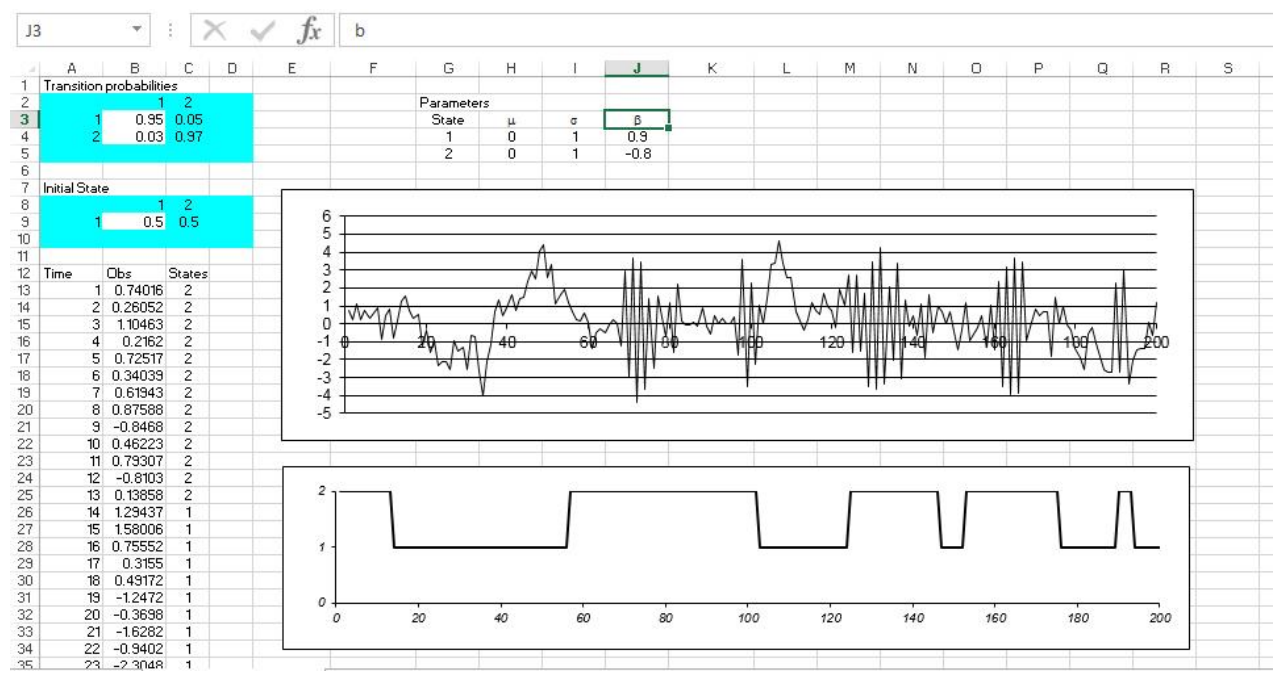

\section{Identification of the State Sequence From the Data Sequence for an Autoregressive Hidden Markov (ARHMM) Model}

The Forward and Backward algorithm described below is a special case of Kalman Filtering (Elliott, Aggoun \& Moore, 1995).

\subsection{The Forward Method for an Autoregressive Hidden Markov Model}

Let $\mathbf{Y}^{(t)}=\left(Y_{1}, Y_{2}, \ldots, Y_{t}\right)^{\prime}$ and $\mathbf{y}^{(t)}=\left(y_{1}, y_{2}, \ldots, y_{t}\right)^{\prime}$

Consider

$\alpha_{t}\left(i_{t}\right)=P\left[\boldsymbol{Y}^{(t)}=\boldsymbol{y}^{(t)}, X_{t}=i_{t},\right]=P\left[Y_{1}=y_{1}, Y_{2}=y_{2}, \ldots, Y_{t}=y_{t}, X_{t}=i_{t}\right]$

Now

$$
\begin{aligned}
& \alpha_{1}\left(i_{1}\right)=P\left[\boldsymbol{Y}^{(1)}=\boldsymbol{y}^{(1)}, X_{1}=i_{1}\right] \\
& =P\left[X_{1}=i_{1}\right] P\left[Y_{1}=y_{1} \mid X_{1}=i_{1}\right] P\left[X_{2}=i_{2} \mid X_{1}=i_{1}\right] P\left[Y_{2}=y_{2} \mid Y_{1}=y_{1}, X_{2}=i_{2} X_{1}=i_{1}\right] \\
& =\pi_{i_{1}} b_{i_{1} y_{1}} g_{i_{2} i_{1}} b_{y_{2} \mid Y_{1}=y_{1}, X_{2}=i_{2}, X_{1}=i_{1}} \\
& \alpha_{t}\left(i_{t}\right)=P\left[\boldsymbol{Y}^{(t)}=\boldsymbol{y}^{(t)}, X_{t}=i_{t}\right] \\
& =\sum_{i_{t-1}} P\left[\boldsymbol{Y}^{(t-1)}=\boldsymbol{y}^{(t-1)}, Y_{t}=y_{t}, X_{t}=i_{t}, X_{t-1}=i_{t-1}\right] \\
& =\sum_{i_{t-1}} P\left[\boldsymbol{Y}^{(t-1)}=\boldsymbol{y}^{(t-1)}, X_{t-1}=i_{t-1}\right] P\left[X_{t}=i_{t} \mid X_{t-1}=i_{t-1}\right] P\left[Y_{t}\right. \\
& \left.=y_{t} \mid X_{t}=i_{t}, X_{t-1}=i_{t-1}, Y_{t-1}=y_{t-1}\right] \\
& =\sum_{\mathrm{i}_{\mathrm{t}-1}} \mathrm{~g}_{\mathrm{i}_{\mathrm{t}} \mathrm{i}_{\mathrm{t}-1}} \alpha_{\mathrm{t}-1}\left(\mathrm{i}_{\mathrm{t}-1}\right) \mathrm{b}_{\mathrm{y}_{\mathrm{t}} \mid \mathrm{Y}_{\mathrm{t}-1}=\mathrm{y}_{\mathrm{t}-1}, \mathrm{x}_{\mathrm{t}}=\mathrm{i}_{\mathrm{t}}, \mathrm{x}_{\mathrm{t}-1}=\mathrm{i}_{\mathrm{t}-1}}=\sum_{\mathrm{i}_{\mathrm{t}-1}} \mathrm{~A}\left(\mathrm{i}_{\mathrm{t}-1}, \mathrm{i}_{\mathrm{t}}\right) \alpha_{\mathrm{t}-1}\left(\mathrm{i}_{\mathrm{t}-1}\right)
\end{aligned}
$$

where $A\left(i_{t-1}, i_{t}\right)=g_{i_{t} i_{t-1}} b_{y_{t} \mid Y_{t-1}=y_{t-1}, X_{t}=i_{t}, X_{t-1}=i_{t-1}}$

and $b_{y_{t} \mid Y_{t-1}=y_{t-1}, X_{t}=i, X_{t-1}=j}$ is the normal density Normal density with

mean $\mu_{\mathrm{i}}+\beta_{\mathrm{i}}\left(\frac{\mathrm{y}_{\mathrm{t}-1}-\mu_{\mathrm{j}}}{\sigma_{\mathrm{j}}}\right)=\mathrm{a}_{\mathrm{i}}^{\mathrm{j}}+\mathrm{b}_{\mathrm{i}}^{\mathrm{j}} \mathrm{y}_{\mathrm{t}-1}$ and standard deviation $\sigma_{i}$.

Also $a_{i}^{j}=\mu_{i}-\left(\frac{\beta_{i}}{\sigma_{j}}\right) \mu_{j}$ and $b_{i}^{j}=\frac{\beta_{i}}{\sigma_{j}}$. 
The forward values $\alpha_{t}(1)$ and $\alpha_{t}(2)$ can be calculated in excel as follows:

1. Place the sequence 2,3, 4 in the cells D1:D3 and in the cells D4:D6

2. Place the formula " $=\mathrm{VLOOKUP}(\mathrm{E} \$ 12, \$ \mathrm{~K} \$ 4: \$ N \$ 5, \$ \mathrm{D} 1)$ " in cell $\mathrm{E} 1$. This formula is then copied to the cells E1:H3.

3. Place the formula "=VLOOKUP $(\mathrm{E} \$ 13, \$ \mathrm{~K} \$ 4: \$ N \$ 5, \$ D 1) "$ in cell E4. This formula is then copied to the cells E4:H6.

This places the parameters of the two successive states $(,$, ) in the cells E1:H6

The values of $a_{i}^{j}=\mu_{i}-\left(\frac{\beta_{i}}{\sigma_{j}}\right) \mu_{j}$ and $b_{i}^{j}=\frac{\beta_{i}}{\sigma_{j}}$ are calculated by placing the formula " $=\mathrm{E} 3 / \mathrm{E} 5$ " in cell E9 to compute $b_{i}^{j}=\frac{\beta_{i}}{\sigma_{j}}$ and the formula "=E1-E9*E4" in the cell E8 to compute $a_{i}^{j}=\mu_{i}-$ $\left(\frac{\beta_{i}}{\sigma_{j}}\right) \mu_{j}$ The formulae in cells E8:E9 are copied to the range E8:H9 to compute of $a_{i}^{j}$ and $b_{i}^{j}$ for other combinations of the state sequence $(i, j)$.

4. Place the formula " $=\mathrm{E} \$ 10 * \mathrm{NORMDIST}(\$ \mathrm{~B} 15, \mathrm{E} \$ 8+\mathrm{E} \$ 9 * \$ \mathrm{~B} 14, \mathrm{E} \$ 2, \mathrm{FALSE}) "$ in cell E15 to compute $A\left(i_{t-1}, i_{t}\right)=g_{i_{t} i_{t-1}} b_{y_{t} \mid Y_{t-1}=y_{t-1}, X_{t}=i_{t}, X_{t-1}=i_{t-1}}$ for $\left(i_{t-1}, i_{t}\right)=(1,1)$. Copy the formula in cell E15 to the Cells E15:H214 to compute $A\left(i_{t-1}, i_{t}\right)$ for $\left(i_{t-1}, i_{t}\right)=(1,1),(2,1),(1,2)$ and $(2,2)$ from $t$ $=1, \ldots, 200$.

5. Place the formula "=B9*NORMDIST(B15,E1,E2,FALSE)" in the cell I15 and the formula "=C9*NORMDIST(B15,H4,H5,FALSE)" in cell J15, to compute $\alpha_{1}(1)$ and $\alpha_{1}(2)$.

6. Place the formulae "=SUMPRODUCT(E16:F16,I15:J15)" and "=SUMPRODUCT(G16:H16,I15:J15)" in the cells I16 and J16. This is the iterative step of the forward algorithm.

The formulae in cells I16:J16 are copied to cells I16:J214. This computes the calculation of $\alpha_{t}(1)$ and $\alpha_{t}(2)$ for $t=1,2, \ldots, 200$

4.2 The Backward Method for a Autoregressive Hidden Markov Model

$$
\begin{aligned}
& \text { Let } \mathbf{Y}^{*(\boldsymbol{t})}=\left(Y_{\boldsymbol{t}+1}, Y_{t+2}, \ldots, Y_{T}\right)^{\prime} \text { and } \mathbf{y}^{*(\boldsymbol{t})}=\left(y_{t+1}, y_{t+2}, \ldots, y_{T}\right)^{\prime} \\
& \text { Consider } \alpha_{t}^{*}\left(i_{t}\right)=P\left[\mathbf{Y}^{*(\boldsymbol{t})}=\mathbf{y}^{*(t)} \mid X_{t}=i_{t}, Y_{t}=y_{t}\right] \\
& =P\left[Y_{t+1}=y_{t+1}, Y_{t+2}=y_{t+2}, \ldots, Y_{T}=y_{T} \mid X_{t}=i_{t}, Y_{t}=y_{t}\right] \\
& \text { Note } \alpha_{T-1}^{*}\left(i_{T-1}\right)=P\left[\mathbf{Y}^{*(\boldsymbol{T}-\mathbf{1})}=y^{*(T-1)} \mid X_{T-1}=i_{T-1}, Y_{T-1}=y_{T-1}\right] \\
& =P\left[Y_{T}=y_{T} \mid X_{T-1}=i_{T-1}, Y_{T-1}=y_{T-1}\right] \\
& =\sum_{i_{T}} P\left[Y_{T}=y_{T}, X_{T}=i_{T} \mid X_{T-1}=i_{T-1}, Y_{T-1}=y_{T-1}\right] \\
& =\sum_{\boldsymbol{i}_{\boldsymbol{T}}} \boldsymbol{\gamma}_{\boldsymbol{i}_{\boldsymbol{T}-1} \boldsymbol{i}_{\boldsymbol{T}}} b_{y_{T} \mid Y_{T-1}=y_{T-1}, X_{T-1}=i_{T-1}, X_{T}=i_{T}} \\
& \text { Now } \alpha_{t}^{*}\left(i_{t}\right)=P\left[\mathbf{Y}^{*(\boldsymbol{t})}=\mathbf{y}^{*(t)} \mid X_{t}=i_{t}, Y_{t}=y_{t}\right] \\
& =P\left[Y_{t+1}=y_{t+1}, \mathbf{Y}^{*(\boldsymbol{t}+\mathbf{1})}=\mathbf{y}^{*(\boldsymbol{t}+\mathbf{1})} \mid X_{t}=i_{t}, Y_{t}=y_{t}\right] \\
& =\sum_{i_{t+1}} P\left[Y_{t+1}=y_{t+1}, Y^{*(t+1)}=y^{*(t+1)}, X_{t+1}=i_{t+1} \mid X_{t}=i_{t}, Y_{t}=y_{t}\right] \\
& =\sum_{i_{t+1}} P\left[Y_{t+1}=y_{t+1}, X_{t+1}=i_{t+1} \mid X_{t}=i_{t}, Y_{t}=y_{t}\right] \times \\
& P\left[\mathbf{Y}^{*(\boldsymbol{t}+\mathbf{1})}=\mathbf{y}^{*(\boldsymbol{t}+\mathbf{1})} \mid Y_{t+1}=y_{t+1}, X_{t}=i_{t}, Y_{t}=y_{t}, X_{t+1}=i_{t+1}\right]
\end{aligned}
$$




$$
\begin{aligned}
= & \sum_{i_{t+1}} P\left[Y_{t+1}=y_{t+1}, X_{t+1}=i_{t+1} \mid X_{t}=i_{t}, Y_{t}=y_{t}\right] \times P\left[\mathbf{Y}^{*(\boldsymbol{t}+\mathbf{1})}=\mathbf{y}^{*(\boldsymbol{t}+\mathbf{1})} \mid Y_{t+1}=y_{t+1}, X_{t+1}=i_{t+1}\right] \\
= & \sum_{i_{t+1}} P\left[X_{t+1}=i_{t+1} \mid X_{t}=i_{t}\right] P\left[Y_{t+1}=y_{t+1} \mid X_{t}=i_{t}, Y_{t}=y_{t}\right] \\
& \times P\left[\mathbf{Y}^{*(\boldsymbol{t}+\mathbf{1})}=\mathbf{y}^{*(\boldsymbol{t}+\mathbf{1})} \mid Y_{t+1}=y_{t+1}, X_{t+1}=i_{t+1}\right] \\
= & \sum_{i_{t+1}} \alpha_{t+1}^{*}\left(i_{t+1}\right) \gamma_{i_{t} i_{t+1}} b_{y_{t+1} \mid Y_{t}=y_{t}, X_{t+1}=i_{t+1}, X_{t}=i_{t}}=\sum_{i_{t+1}} \alpha_{t+1}^{*}\left(i_{t+1}\right) A\left(i_{t}, i_{t+1}\right)
\end{aligned}
$$

where $A\left(i_{t}, i_{t+1}\right)=g_{i_{t} i_{t+1}} b_{y_{t+1} \mid Y_{t}=y_{t}, X_{t+1}=i_{t+1}, X_{t}=i_{t}}$

Also $b_{y_{t+1} \mid Y_{t}=y_{t}, X_{t+1}=j, X_{t}=i}$ is the normal density Normal density

with mean $\mu_{j}+\beta_{j}\left(\frac{y_{t}-\mu_{i}}{\sigma_{i}}\right)=a_{i}^{j}+b_{i}^{j} y_{t}$ and standard deviation $\sigma_{i}$.

Also $a_{i}^{j}=\mu_{j}-\left(\frac{\beta_{j}}{\sigma_{i}}\right) \mu_{i}$ and $b_{i}^{j}=\frac{\beta_{j}}{\sigma_{i}}$

The backward values $\alpha_{t}^{*}(1)$ and $\alpha_{t}^{*}(2)$ can be calculated in excel as follows:

1. Place the formula "=SUM(E214:F214)" in the cell K214 and the formula "=SUM(G214:H214)" in cell L214, to compute $\alpha_{199}^{*}(1)$ and . $\alpha_{199}^{*}(2)$

2. Place the formulae "=SUMPRODUCT(E213:F213,K214:L214)" and

"=SUMPRODUCT(G213:H213,K214:L214)" in the cells K213 and L213. This is the iterative step of the backward algorithm.

The formulae in cells K213:L213 are copied to cells K16:L213. This computes the calculation of $\alpha_{t}^{*}(1)$ and.$\alpha_{t}^{*}(2) \alpha_{t}(1)$ and $\alpha_{t}(2)$ for $t=1,2, \ldots, 200$

\section{Calculation of ARHMM State Probabilities Given the Data Sequence $Y$}

Now recall that

$\alpha_{t}\left(i_{t}\right)=P\left[\boldsymbol{Y}^{(t)}=\boldsymbol{y}^{(t)}, X_{t}=i_{t}\right]=P\left[Y_{1}=y_{1}, Y_{2}=y_{2}, \ldots, Y_{t}=y_{t}, X_{t}=i_{t}\right]$

and $\alpha_{t}^{*}\left(i_{t}\right)=P\left[\mathbf{Y}^{*(t)}=\mathbf{y}^{*(t)} \mid X_{t}=i_{t}, Y_{t}=y_{t}\right]$

$$
=P\left[Y_{t+1}=y_{t+1}, Y_{t+2}=y_{t+2}, \ldots, Y_{T}=y_{T} \mid X_{t}=i_{t}, Y_{t}=y_{t}\right]
$$

Thus $\alpha_{t}\left(i_{t}\right) \alpha_{t}^{*}\left(i_{t}\right)=P\left[\boldsymbol{Y}=\boldsymbol{y}, X_{t}=i_{t},\right]$

And $P[\boldsymbol{Y}=\boldsymbol{y}]=\sum_{i_{t}} P\left[\boldsymbol{Y}=\boldsymbol{y}, X_{t}=i_{t}\right]=\sum_{i_{t}} \alpha_{t}\left(i_{t}\right) \alpha_{t}^{*}\left(i_{t}\right)$

Thus $P\left[X_{t}=i_{t}\lfloor\boldsymbol{Y}=\boldsymbol{y}]=\frac{P\left[\boldsymbol{Y}=\boldsymbol{y}, X_{t}=i_{t}\right]}{P[\boldsymbol{Y}=\boldsymbol{y}]}=\frac{\alpha_{t}\left(i_{t}\right) \alpha_{t}^{*}\left(i_{t}\right)}{\sum_{i_{t}} \alpha_{t}\left(i_{t}\right) \alpha_{t}^{*}\left(i_{t}\right)}\right.$

and $P\left[X_{t}=2[\boldsymbol{Y}=\boldsymbol{y}]=\frac{\alpha_{t}(2) \alpha_{t}^{*}(2)}{\sum_{i_{t}} \alpha_{t}\left(i_{t}\right) \alpha_{t}^{*}\left(i_{t}\right)}\right.$

The conditional probabilities are calculated as follows

Place the formula " $=\mathrm{J} 15 * \mathrm{~L} 15 /(\mathrm{J} 15 * \mathrm{~L} 15+\mathrm{I} 15 * \mathrm{~K} 15)$ " in cell D15.

Copy the formula in C15 to cells C15:C214

Graphs that now can be plotted are $t$ vs $y_{t}$ (observation), $t$ vs $x_{t}$ (state) and $P\left[X_{t}=2[\boldsymbol{Y}=\boldsymbol{y}]\right.$ 

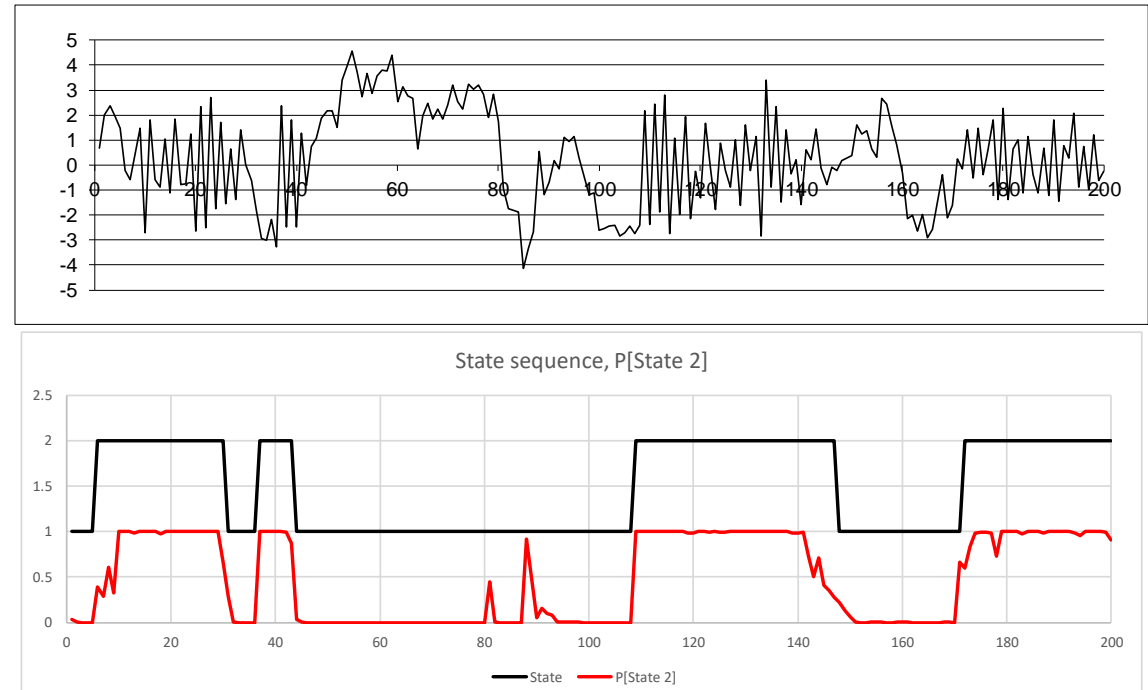

\section{Exercises That Can be Performed to Illustrate the Performance of the Backward and Forward Algorithm for ARHMM Series}

In these exercises we generate realizations using different sets of parameters to examine the performance of Forward and Backward algorithms in identifying the hidden states of an Auto regressive Hidden Markov model.

1. $\mu_{1}=10, \sigma_{1}=1, \beta_{1}=.95, \mu_{2}=5, \sigma_{2}=1, \beta_{2}=-.95$
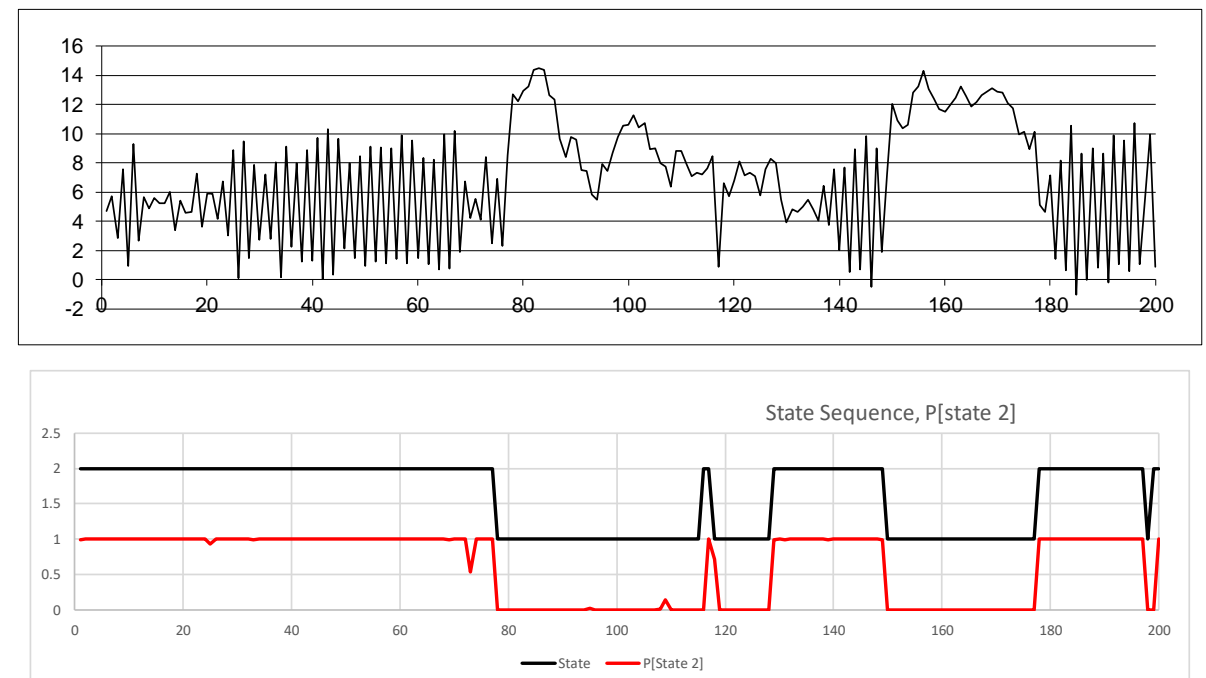

Comment: Differing means, equal and small standard deviations, auto correlations large in absolute value and differing in sign, resulting in accurate prediction of states from data.

2. $\mu_{1}=5, \sigma_{1}=1, \beta_{1}=.95, \mu_{2}=5, \sigma_{2}=1, \beta_{2}=-.95$

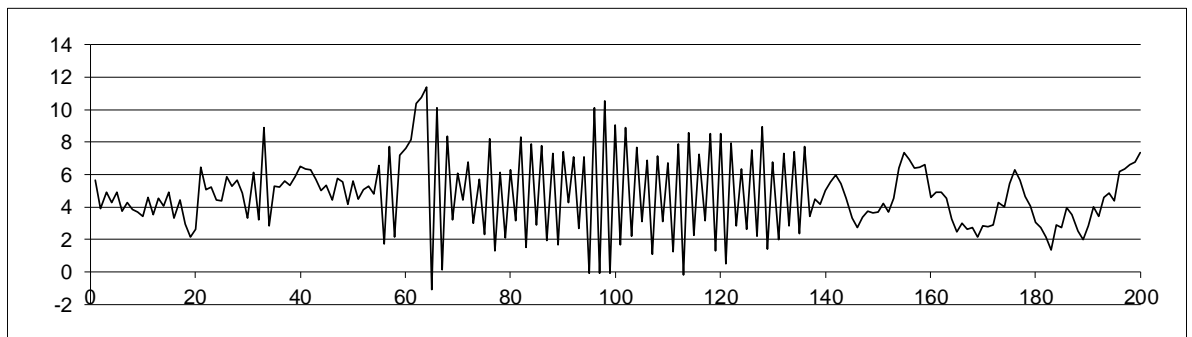




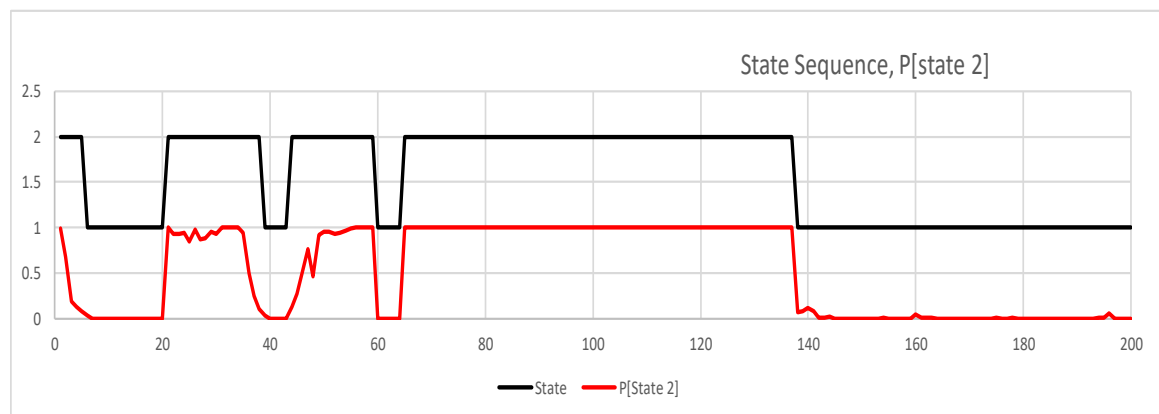

Comment: Equal means, equal and small standard deviations, auto correlations large in absolute value and differing in sign, also resulting in accurate prediction of states from data.

3. $\mu_{1}=5, \sigma_{1}=1, \beta_{1}=.45, \mu_{2}=5, \sigma_{2}=1, \beta_{2}=-.45$ )
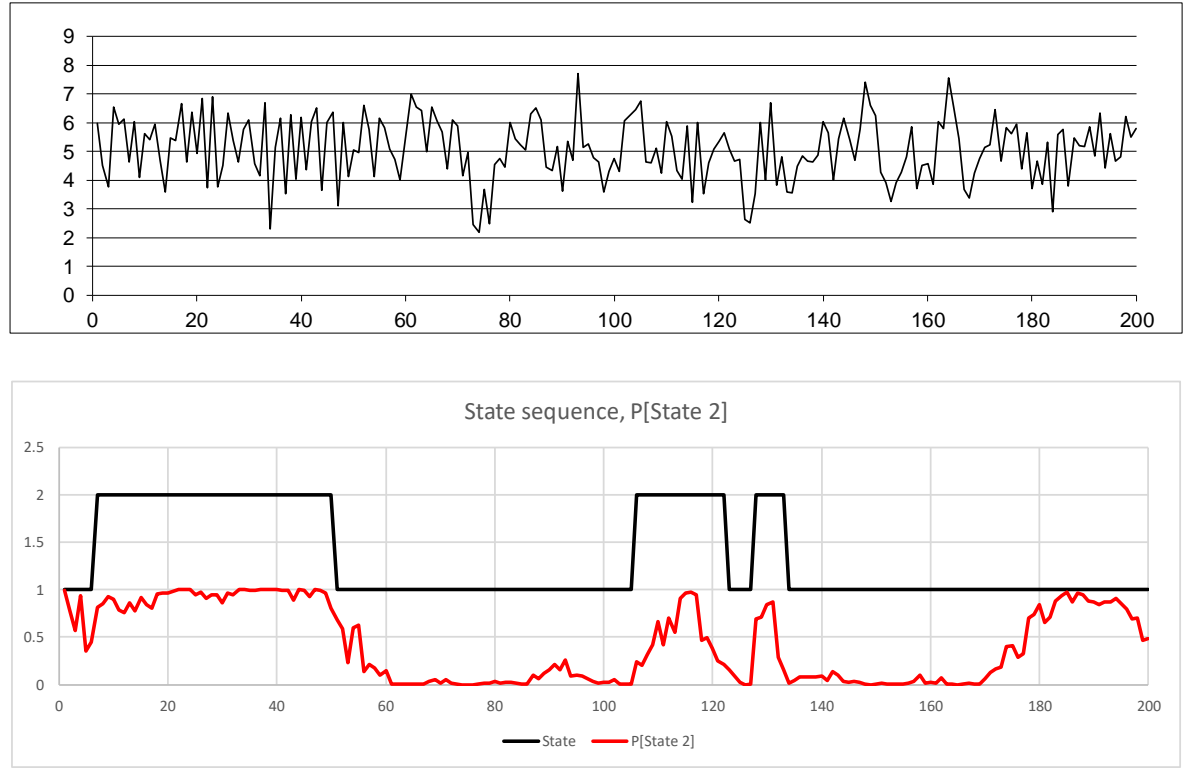

Comment: Equal means, equal and small standard deviations, auto correlations medium in absolute value and differing in sign, also resulting in less accurate prediction of states from data.

4. $\mu_{1}=5, \sigma_{1}=1, \beta_{1}=.25, \mu_{2}=5, \sigma_{2}=1, \beta_{2}=-.25$ )
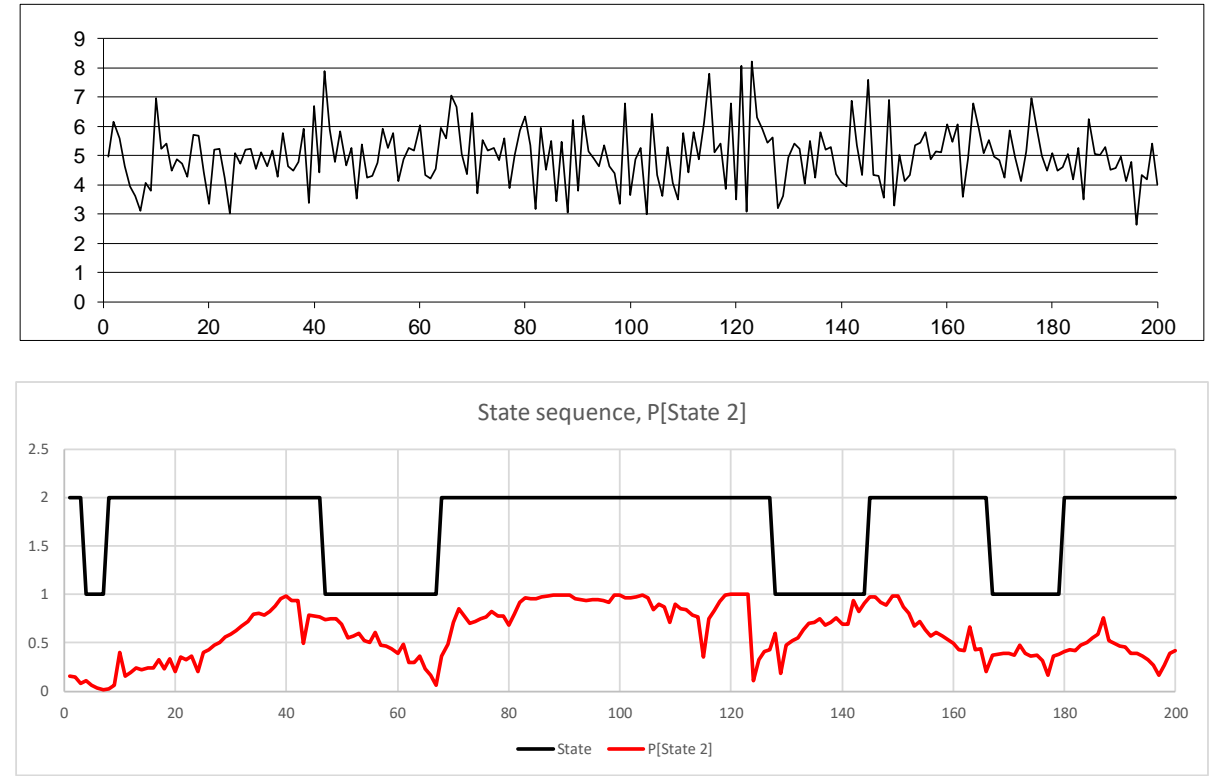
Comment: Accuracy of state identification decreases as absolute value of autocorrelations decrease in value.

5. $\left.\mu_{1}=5, \sigma_{1}=1, \beta_{1}=.95, \mu_{2}=5, \sigma_{2}=1, \beta_{2}=.95\right)$



Comment: When the parameters are the same for both states the data provides no distinguishing information. The Forward and Backward algorithm gives the long run state distribution for the unseen Markov chain.

\section{Conclusion}

Auto-Regressive Hidden Markov models (ARHMM) are increasingly being used by researchers in a variety of disciplines to identify underlying states with correlated observations contributing to patterns in observed data. For example, Tang (2004) used Auto-Regressive Hidden Markov models on temperature data in the Pacific Ocean to study the mechanism of El Nino. Stanculescu, Williams, and Freer (2014) used Auto-Regressive Models for the early detection of neo-natal sepsis, one of the major clinical concerns when premature babies receive intensive care. This approach gives earlier detection of sepsis than the slower laboratory tests that are currently used for this condition. More recently, Dang, Chaudhuty, Lall and Roy (2017) used an autoregressive model to provide effective connectivity estimates among brain states using fMRI signals. These hidden states can provide new information about a process over time that may be missed by traditional statistical approaches. To this end, DeGeorgia, Kaffashi, Jacono and Loparo (2015) point out that the medical field has not yet incorporated new advances in computer science, mathematics, and allied fields regarding the recent increased capability in data collection for patient monitoring. We would also add that Auto-Regressive Hidden Markov models have potential in intensive care monitoring and the analysis of medical data.

The purpose of this article was to demonstrate how the Forward and Backward algorithm performs in ARHMM state identification using Excel. By using this article and the included exercises one can develop an applied understanding of underlying state identification in the Auto-Regressive Hidden Markov Model. While only two-state ARHMM was considered in this article, more can be considered with minor modifications to the EXCEL file.

\section{Disclosure Statement}

The authors declare no conflict of interest.

\section{References}

Bing-Hwang, J., \& Rabiner, L. R. (1986). Mixture Autoregressive Hidden Markov Models for speech signal. IEEE Transactions on Acoustics Speech and Signal Processing, 33, 1404-1413. https://doi.org/10.1109/TASSP.1985.1164727

Dang, S., Chaudhury, S., \& Lall, S., \& Roy, P. K. (2017). Learning effective connectivity from fMRI using autoregressive Hidden Markov Model with missing data. Journal of Neuroscience Methods, 278(2017), 87-100. https://doi.org/10.1016/j.jneumeth.2016.12.019

DeGeorgia, D., Kaffashi, F., Jacono, J., \& Loparo, K. A. (2015). Information technology in critical care: review of monitoring and data acquisition systems for data care and research. The Scientific World Journal, Article ID: 727694. https://doi.org/10.1155/2015/727694

Elliott, R. J., Aggoun, L., \& Moore, J. B. (1995). Hidden Markov Models, Springer, Switzerland. 
Fishman, G. S. (1995). Monte Carlo, Concepts, Algorithms and Applications, Springer Switzerland.

Genon-Catalot, V. T., Jeantheau, T., \& Laredo, C. (2000). Stochastic volatility models as Hidden Markov Models and Statistical applications, Bernoulli, 6, 1051-1079. https://doi.org/10.2307/3318471

Laverty, W. H., \& Kelly, I. W (2019). Using Excel to visualize state identification in Hidden Markov Models using the forward and backward algorithms, Applied Mathematical Sciences, 13, 151-162. https://doi.org/10.12988/ams.2019.812195

Laverty, W. H., Miket, M. J., \& Kelly, I. W. (2002 a). Application of hidden Markov models on residuals: an example using Canadian traffic accident data, Perceptual and Motor Skills, 94(3 Pt 2), 1151-6. https://doi.org/10.2466/pms.2002.94.3c.1151

Laverty, W. H., Miket, M. J., \& Kelly, I. W. (2002b). Examination of residuals to Vancouver crisis call data by using Hidden Markov Models, Perceptual and Motor Skills, 94, 548-550. https://doi.org/10.2466/pms.2002.94.2.548

Laverty, W. H., Miket, M. J., \& Kelly, I. W. (2002c). Simulation of hidden Markov models with EXCEL, Journal of Royal Statistical Society: Series D, 51, 31-40. https://doi.org/10.1111/1467-9884.00296

Mamon, R., \& Elliott, R. (2007). Hidden Markov Models in Finance. In Operations Research and Management Science, Springer, Switzerland. https://doi.org/10.1007/0-387-71163-5

Rabiner, L. (1989). A tutorial on hidden markov models and selected applications in speech recognition, Proceedings of the IEEE, 77, 257 -286. https://doi.org/10.1109/5.18626

Stanculescu, C. K., Williams, I., \& Freer, Y. (2014). Autoregressive Hidden Markov Models for the Early Detection of Neonatal Sepsis. IEEE Journal of Biomedical and Health Informatics, 18, 1560-1570. https://doi.org/10.1109/JBHI.2013.2294692

Tang, X. (2004). Autoregressive Hidden Markov Model with Application in an El Niño study, Master's thesis, University of Saskatchewan, Saskatoon, Saskatchewan, Canada.

Walker II, M. (2011). Hidden Markov Models for Heart Rate Variability with Biometric Applications, All Theses and Dissertations (ETDs). 491.

\section{Copyrights}

Copyright for this article is retained by the author(s), with first publication rights granted to the journal.

This is an open-access article distributed under the terms and conditions of the Creative Commons Attribution license (http://creativecommons.org/licenses/by/4.0/). 